\title{
Co-localization of GSTP1 and JNK in transitional cell carcinoma of urinary bladder
}

Marija Pljesa-Ercegovac ${ }^{1}$, Ana Savic-Radojevic ${ }^{1}$, Tamara Kravic-Stevovic ${ }^{2}$, Vladimir Bumbasirevic ${ }^{2}$, Jasmina Mimic-Oka ${ }^{1}$ and Tatjana Simic ${ }^{1}$

${ }^{1}$ Faculty of Medicine, Institute of Medical and Clinical Biochemistry, University of Belgrade, Belgrade, Serbia.

${ }^{2}$ Faculty of Medicine, Institute of Histology and Embryology, University of Belgrade, Belgrade, Serbia.

\begin{abstract}
Transitional cell carcinoma (TCC) of urinary bladder belongs to glutathione S-transferase P1 (GSTP1) overexpressing tumors. Upregulated GSTP1 in TCC is related to apoptosis inhibition. This antiapoptotic effects of GSTP1 might be mediated through protein:protein interaction with c-Jun $\mathrm{NH}_{2}$-terminal kinase (JNK). Herein, we analyzed whether a direct link between GSTP1 and JNK exists in TCC. The presence of GSTP1/JNK complexes was analyzed by immunoprecipitation and Western blotting in 20 TCC specimens, obtained after surgery. Co-localization of GSTP1 and JNK was also investigated in the 5637 TCC cell line by immunofluorescence confocal microscopy. By means of immunoprecipitation we show for the first time the presence of GSTP1/JNK complexes in all TCC samples studied. A co-localization of GSTP1 and JNK was also demonstrated in the 5637 TCC cell line by means of confocal microscopy. Protein-protein interactions, together with co-localization between GSTP1 and JNK provide evidence that GSTP1 most probably inhibits apoptosis in TCC cells by non-covalent binding to JNK.
\end{abstract}

Key words: glutathione S-transferase, JNK, TCC, co-localization.

Received: November 11, 2009; Accepted: May 14, 2010.

The glutathione transferases are a multigene family of isozymes that catalyze the nucleophilic attack of the sulfur atom of glutathione on electrophilic groups of substrate molecules (Hayes and Strange, 2000). Glutathione transferase P1 (GSTP1) is the most prevalent in mammalian cells (Townsend and Tew, 2003). GSTP1 is overexpressed in many tumors, including transitional cell carcinoma (TCC) of urinary bladder, where its activity and expression correlate with tumor stage and grade (Berendsen et al., 1997; Townsend and Tew, 2003; Simic et al., 2005). Because of the defined role of GST in drug metabolism, elevated expression of GSTP1 in tumors has been frequently associated with detoxification reactions. Nevertheless, GSTP1 overexpression has been found in drug resistant cells, even in instances where there is no evidence that the selecting drug is a substrate for GSTP1 (Gate and Tew, 2001; Townsend and Tew, 2003). Recently, a new insight into a functional link between upregulated GSTP1 and the malignant phenotype has been suggested from growing evidence that GSTs are also involved in the regulation of stress signaling and resistance to apoptosis by mechanisms

Send correspondence to Marija Pljesa-Ercegovac. Faculty of Medicine, Institute of Medical and Clinical Biochemistry, University of Belgrade, Pasterova 2, 11000 Belgrade, Serbia. E-mail: marijaercegovac@med.bg.ac.rs. independent of their catalytic activity; this regulatory role depending on cellular redox status (Wang et al., 2001; Adler and Pincus, 2004; Simic et al., 2009). In TCC, the oxidant-antioxidant balance favors the reduced state, as increased levels of glutathione, the major cellular nonprotein antioxidant, together with upregulated antioxidant enzymes have been observed in this setting (Yang et al., 1997; Savic-Radojevic et al., 2007). High intracellular thiol levels and the absence of oxidative stress promote the existence of GSTP1 in monomeric form, while catalitically active GSTP1 is normally dimerized. Redox-active monomeric GSTP1 subunits inhibit c-Jun $\mathrm{NH}_{2}$-terminal kinase (JNK), an enzyme that triggers the apoptotic cascade in several cancer cell lines (Wang et al., 2001). From our point of view, high antioxidant capacity also promotes an antiapoptotic role of GSTP1 in TCC. In favor of such a hypothesis are recent data showing a significant negative correlation between GSTP1 and cleaved caspase 3 expression in human TCC specimens (Pljesa-Ercegovac et al., 2009). Nevertheless, the direct link between GSTP1 and JNK in TCC still has to be confirmed. Herein, we analyzed the presence of GSTP $1 / \mathrm{JNK}$ complexes in specimens of tumor tissue of the urinary bladder obtained from 20 patients with TCC after radical cystectomy, as well as in the 5637 TCC cell line. Specimens of tumor tissue were taken in the oper- 
ating theatre in the presence of a clinical pathologist who performed the histopathology examination. All patients gave informed consent to enter the study. The ethics committee approved the use of human tissue for research.

Tumor samples were washed in cold saline, frozen in liquid nitrogen and stored at $-80{ }^{\circ} \mathrm{C}$ until use. Immunoprecipitation experiments were performed using the primary antibody against JNK (Sigma-Aldrich, St. Louis, USA) and the Protein A-agarose (Roche Applied Science, Mannheim, Germany). Samples were homogenized with the lysis buffer provided by the manufacturer and centrifuged at $100.000 \mathrm{x}$ g for $45 \mathrm{~min}$ at $4{ }^{\circ} \mathrm{C}$. Cytosolic fractions of TCC specimens were incubated with $2 \mu \mathrm{L}$ of anti-JNK antibody overnight at $4{ }^{\circ} \mathrm{C}$. Immunoblots were then probed with the anti-GSTP1 antibody (Sigma-Aldrich, St. Louis, USA). JNK expression was determined on the same membrane after stripping off the immune complex for the detection of GSTP1. Immunoblot analysis showed an absence of non-specific binding of the JNK antibody to GSTP1. Control immunoprecipitations, that were performed in the absence of anti-JNK antibody, ruled out possible unspecific pull-down of GSTP1. Confocal microscopy on cells of the 5637 cell line was performed using anti-GSTP1 antibody followed by FITC-conjugated secondary antibody (Dako, Glostrup, Danmark), as well as anti-JNK antibody followed by TRITC-conjugated secondary antibody (Dako, Glostrup, Danmark). Coverslips were mounted with fluorescent mounting medium (Dako, Glostrup, Danmark), observed and photographed under confocal scanning mi- croscope (Leica LCS). The specificity of the primary antibodies used was previously confirmed by Western blot analysis. Control experiments for non-specific binding were performed in parallel by omission of the primary antibody.

Immunoprecipitation, followed by Western blotting showed the presence of GSTP1/JNK complexes in all TCC samples studied (Figure 1A). Furthermore, co-localization of GSTP1 and JNK was confirmed in 5637 TCC cell line by means of immunofluorescence confocal microscopy (Figure 1B). These results are in agreement with data of Adler and Pincus (2004) who performed molecular dynamics investigations on the 3-dimensional structure of GSTP1, free and bound to an inhibitor, that blocks its ability to inhibit JNK activation (Adler and Pincus, 2004). Four putative domains are involved in the interaction between GSTP1 and JNK. Two of these are involved in GSTP1 binding to JNK, whereas the other two affect phosphorylation of JNK. The proposed mechanism by which GSTP1 inhibits activation of JNK is by either blocking phosphorylation of JNK or by promoting dephosphorylation of phosphorylated JNK (Adler and Pincus, 2004). In this manner, JNK is prevented from activating downstream targets in the apoptotic pathway, such as c- Jun. Our results support such a hypothesis, because the activated JNK signal was not detected in our immunoprecipitation experiments.

The presence of GSTP1/JNK complex has also been reported in human leukemia, hepatic carcinoma and neuroblastoma cells (Ricci et al., 2005; Turella et al., 2005; Cui

(B)

(A)

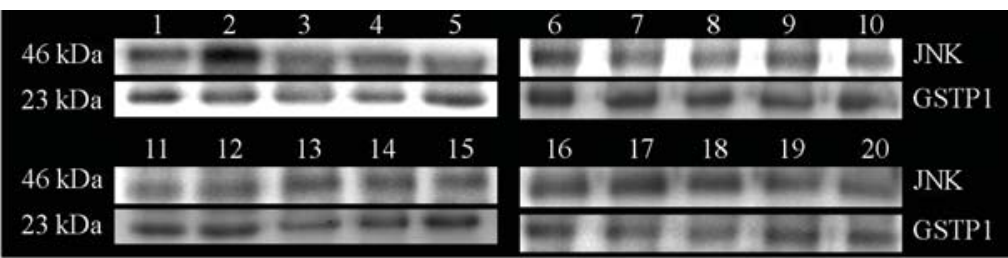

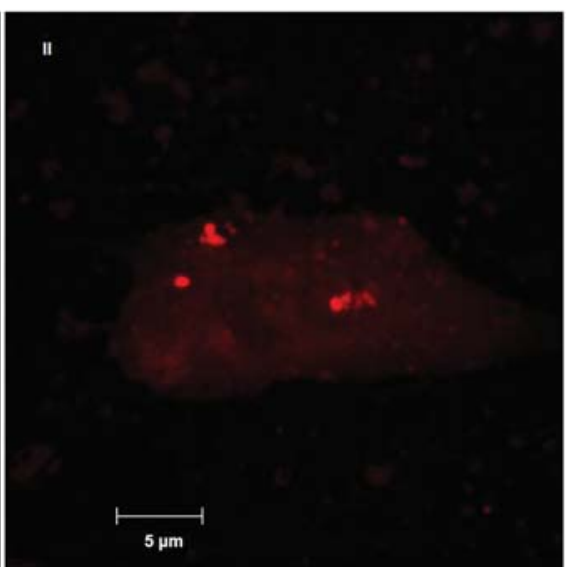

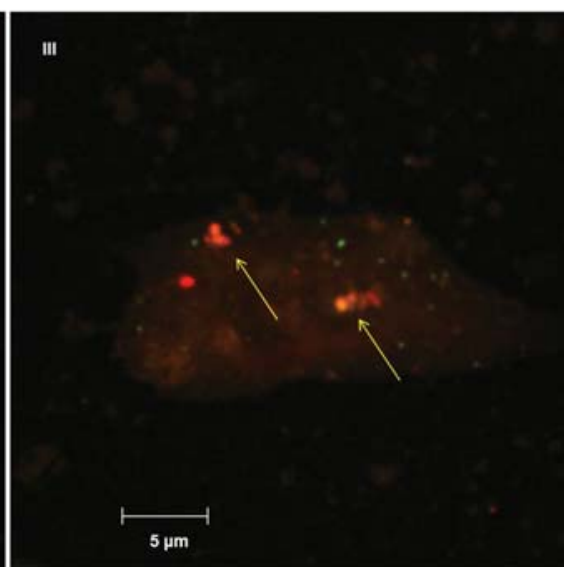

Figure 1 - (A) Co-immunoprecipitation of GSTP1 with JNK in human TCC specimens. Immunoprecipitation was performed using protein A-agarose to retain JNK/GSTP1 complexes. Anti-JNK antibody and polyclonal anti-GSTP1 antibody were used for Western blot analysis. (B) Confocal microscopy images of human 5637 TCC cells after incubation with anti-GSTP1 antibody followed by FITC-conjugated secondary antibody (I); after incubation with anti-JNK antibody followed by TRITC-conjugated secondary antibody (II); Image showing co-localization of GSTP1 with JNK (III). Coverslips were mounted with fluorescent mounting medium, observed and photographed under confocal scanning microscope. 
et al., 2008; Castro-Caldas et al., 2009). Therefore, it is possible that in these cells GSTP1 may act as an inhibitor of apoptosis, controlling JNK catalytic activity. In addition to enhancement of tumor progression, upregulated GSTP1 expression in TCC might also limit the efficacy of chemotherapeutic agents that act by inducing apoptosis via the JNK pathway (Wang et al., 2001; Townsend and Tew, 2003; Burg et al., 2006; Lo and Ali-Osman, 2007). To date, several chemotherapeutic agents that are being used in various stages of TCC, such as mytomicine c, adriamycine and cysplatin, induce apoptosis in tumor cells via the JNK pathway. We believe that the presence of GSTP1 in complexes with JNK limits the efficacy of intravesicular and systemic chemotherapy in bladder cancer, affecting patients' survival. In order to improve the response to chemotherapy it seems reasonable that novel small-molecule GSTP1-targeted agents, which were developed to overcome resistance to treatment in ovarian, non-small-cell lung, breast, and colorectal cancers, can also be used to sensitize TCC tumor cells (Tew, 2005; Turella et al., 2005; Vergote et al., 2007).

In conclusion, this study adds to the generality of the interaction between GSTP1 and JNK as a contributing factor to TCC phenotype and might have important implications in the treatment of these tumors.

\section{Acknowledgments}

This work was supported by grants 145009DJ and 145058 from the Serbian Ministry of Science and Technology.

\section{References}

Adler V and Pincus MR (2004) Effector peptides from glutathione S-transferase-pi affect the activation of jun by jun-N-terminal kinase. Ann Clin Lab Sci 34:35-46.

Berendsen CL, Peters WH, Scheffer PG, Bouman AA, Boven E and Newling DW (1997) Glutathione S-transferase activity and subunit composition in transitional cell cancer and mucosa of the human bladder. Urology 49:644-651.

Burg D, Riepsaame J, Pont C, Mulder G and Van de Water B (2006) Peptide bond modified glutathione conjugate analogs modulate GST pi function in glutathione-conjugation, drug sensitivity and JNK signaling. Biochem Pharmacol 71:268277.

Castro-Caldas M, Milagre I, Rodrigues E and Gama MJ (2009) Glutathione S-transferase pi regulates UV-induced JNK signaling in SH-SY5Y neuroblastoma cells. Neurosci Lett 451:241-245.

Cui H, Shen J, Lu D, Zhang T, Zhang W, Sun D and Wang PG (2008) 4-Aryl-1,3,2-oxathiazolylium-5-olate: A novel GST inhibitor to release JNK and activate c-Jun for cancer therapy. Cancer Chemother Pharmacol 62:509-515.

Gate L and Tew KD (2001) Glutathione S-transferases as emerging therapeutic targets. Expert Opin Ther Targets 5:477-489.
Hayes JD and Strange RC (2000) Glutathione S-transferase polymorphisms and their biological consequences. Pharmacology 61:154-166.

Lo HW and Ali-Osman F (2007) Genetic polymorphism and function of glutathione S-transferases in tumor drug resistance. Curr Opin Pharmacol 7:367-374.

Pljesa-Ercegovac M, Savic-Radojevic A, Dragicevic D, MimicOka J, Matic M, Sasic T, Pekmezovic T, Vuksanovic A and Simic T (2009) Enhanced GSTP1 expression in transitional cell carcinoma of urinary bladder is associated with altered apoptotic pathways. Urol Oncol (in press) 10.1016/j. urolonc.2008.10.019.

Ricci G, De Maria F, Antonini G, Turella P, Bullo A, Stella L, Filomeni G, Federici G and Caccuri AM (2005) 7-Nitro2,1,3-benzoxadiazole derivatives, a new class of suicide inhibitors for glutathione S-transferases. J Biol Chem 280:26397-26405.

Savic-Radojevic A, Mimic-Oka J, Pljesa-Ercegovac M, Opacic M, Dragicevic D, Kravic T, Djokic M, Micic S and Simic T (2007) Glutathione S-transferase P1 expression correlates with increased antioxidant capacity in transitional cell carcinoma of the urinary bladder. Eur Urol 52:470-477.

Simic T, Mimic-Oka J, Savic-Radojevic A, Opacic M, Pljesa M, Dragicevic D, Djokic M and Radosavljevic R (2005) Glutathione S-transferase T1-1 activity upregulated in transitional cell carcinoma of urinary bladder. Urology 65:1035-1040.

Simic T, Savic-Radojevic A, Pljesa-Ercegovac M, Matic M and Mimic-Oka J (2009) Glutathione S-transferases in kidney and urinary bladder tumors. Nat Rev Urol 6:281-289.

Tew KD (2005) TLK-286: A novel glutathione S-transferase-activated prodrug. Expert Opin Investig Drugs 14:1047-1054.

Townsend D and Tew K (2003) The role of glutathione S-transferase in anti-cancer drug resistance. Oncogene 22:43694675.

Turella P, Cerella C, Filomeni G, Bullo A, De Maria F, Ghibelli L, Ciriolo MR, Cianfriglia M, Mattei M, Federici G et al. (2005) Proapoptotic activity of new glutathione S-transferase inhibitors. Cancer Res 65:3751-3761.

Vergote I, Finkler N, Del Campo J, Lohr A, Hunter J, Matei D, Spriggs D, Kavanagh J, Vermorken J, Brown GL et al. (2007) Single agent, canfosfamide (C, TLK286) vs. pegylated liposomal doxorubicin (D) or topotecan (T) in 3rd line treatment of platinum $(\mathrm{P})$ refractory or resistant ovarian cancer (OC): Phase 3 study results. J Clin Oncol 25:LBA5528 [Abstract].

Wang T, Arifoglu P, Ronai Z and Tew K (2001) Glutathione S-transferase P1-1 (GSTP1-1) inhibits c-Jun N-terminal kinase (JNK1) signaling through interaction with the C terminus. J Biol Chem 276:20999-21003.

Yang CR, Ou YC, Kuo JH, Kao YL, Chen CL, Yean SY, Horng YY and Yang CS (1997) Intracellular glutathione content of urothelial cancer in correlation to chemotherapy response. Cancer Lett 119:157-162.

Associate Editor: Emmanuel Dias Neto

License information: This is an open-access article distributed under the terms of the Creative Commons Attribution License, which permits unrestricted use, distribution, and reproduction in any medium, provided the original work is properly cited. 